\title{
ALTopt: An R Package for Optimal Experimental Design of Accelerated Life Testing
}

by Kangwon Seo and Rong Pan

\begin{abstract}
The R package ALTopt has been developed with the aim of creating and evaluating optimal experimental designs of censored accelerated life tests (ALTs). This package takes the generalized linear model approach to ALT planning, because this approach can easily handle censoring plans and derive information matrices for evaluating designs. Three types of optimality criteria are considered: $D$-optimality for model parameter estimation, $U$-optimality for reliability prediction at a single use condition, and I-optimality for reliability prediction over a region of use conditions. The Weibull distribution is assumed for failure time data and more than one stress factor can be specified in the package. Several graphical evaluation tools are also provided for the comparison of different ALT test plans.
\end{abstract}

\section{Introduction}

Accelerated life testing (ALT) is commonly used for obtaining a product's failure time data by subjecting it to elevated stress conditions, such as temperature, humidity, and voltage. As a result, the product fails in a shorter time period than would be expected under normal stress conditions. The failure data obtained from ALTs can then be extrapolated to the normal use stress level to estimate the product's lifetime distribution. Nelson $(2005 a, b)$ provides a comprehensive review of ALT papers up to 2005 .

To avoid poor experimental results and to obtain more accurate inference on the acceleration model and on reliability prediction, it is necessary to have an effective ALT test plan. A well-designed ALT test plan often aims to achieve some statistical optimality. However, conventional experimental designs (e.g., factorial designs) are not effective as ALT test plans because of the following features of ALTs:

- Extrapolation - Test stress levels are typically higher than the normal use stress levels. As failure time data will be collected at these higher stress levels, extrapolating them to the normal use stress level is needed for reliability prediction. Nonlinear relationships between failure time and stress levels are expected.

- Non-normal distributions of failure times - The failure time distribution is typically positively skewed, e.g., the Weibull distribution.

- Censoring of failure time data - Censoring occurs when the exact failure times of test units are not observed. There are several reasons for censoring. In some cases, test units do not fail by the end of the test period, in which case the data becomes right-censored. In other cases, test units are periodically inspected, so the only information available is the time interval of failure, while the exact failure time is unknown. The latter case is called interval-censoring.

In this article, we introduce an R package, ALTopt (Seo and Pan, 2015), that constructs optimal test plans for ALTs with right- and interval-censored data. This package is based on the work done by Monroe et al. (2011) and Yang and Pan (2013), where generalized linear models (GLMs) were used to model censored ALT data.

\section{Optimal designs of ALT}

\section{Optimality criteria}

The ALTopt package accommodates three optimality criteria: $D$-optimality, $U$-optimality and $I$ optimality. A $D$-optimal design minimizes the generalized variance of parameter estimates, while a $U$-optimal or I-optimal design minimizes, respectively, the (average) variance of response prediction at a single use condition or over a region of use conditions.

In the GLM context, the $D$-optimal design is defined by

$$
\xi^{*}:=\underset{\xi}{\arg \max }\left|\mathbf{X}(\xi)^{\prime} \mathbf{W X}(\xi)\right|
$$


Let $n$ be the number of test units and $p$ be the number of of model parameters. Matrix $\mathbf{X}(\xi)$ is the $n \times p$ model matrix constructed by expanding a design matrix to include all regression terms in the chosen model form, and matrix $\mathbf{W}$ is the $n \times n$ diagonal matrix of weights that depends on the GLM formulation used.

Using the same notation, the $U$-optimal and $I$-optimal designs can be defined, respectively, as

$$
\xi^{*}:=\underset{\xi}{\arg \min } \mathbf{x}_{u s e}^{\prime} \cdot\left(\mathbf{X}(\xi)^{\prime} \mathbf{W} \mathbf{X}(\xi)\right)^{-1} \cdot \mathbf{x}_{\text {use }},
$$

and

$$
\xi^{*}:=\underset{\xi}{\arg \min } \frac{\int_{\Omega} \mathbf{x}_{u s e}^{\prime} \cdot\left(\mathbf{X}(\xi)^{\prime} \mathbf{W} \mathbf{X}(\xi)\right)^{-1} \cdot \mathbf{x}_{\text {use }} d \mathbf{x}_{u s e}}{S_{\Omega}},
$$

where $\mathbf{x}_{\text {use }}$ is the single use condition, $\Omega$ is the region of use conditions, and $S_{\Omega}$ is the area of use region. For GLMs, the weights $\mathbf{W}$ are functions of the regression coefficients in the linear predictor. Therefore, the information matrix contains unknown model parameter values, implying that the choice of these unknown values also affects the optimal design (see Johnson and Montgomery (2009) for more details). In this article, we assume that these parameter values are pre-specified. They are referred to as the planning values by Meeker and Escobar (1998).

\section{GLMs for ALT}

A function that links failure time and stress variables is needed in order to extrapolate the results obtained in the test region to the use region. The GLM formulation for ALT is built upon the Cox's proportional hazard $(\mathrm{PH})$ assumption. This section provides the derivation of these formulations for right-censored and interval-censored ALT data.

\section{The Cox's proportional hazard model}

The PH model assumes that, given the vector of explanatory variables $\mathbf{x}$, the hazard function of failure time is given by

$$
h(t, \mathbf{x} ; \boldsymbol{\beta})=h_{0}(t) e^{\mathbf{x}^{\prime} \boldsymbol{\beta}},
$$

where $h_{0}(t)$ is called the baseline hazard function and $\beta$ is a vector of regression coefficients.

Note that the baseline hazard function is a function of time only. From Eq. (1) we can derive that

$$
H(t, \mathbf{x})=H_{0}(t) e^{\mathbf{x}^{\prime} \boldsymbol{\beta}},
$$

where $H(t, \mathbf{x})$ is the cumulative hazard function and $H_{0}(t)$ is the baseline cumulative hazard function. It is also easy to show that a reliability function is given by

$$
R(t, \mathbf{x})=\left(R_{0}(t)\right)^{e^{x^{\prime} \beta}}
$$

where $R(t, \mathbf{x})$ is the reliability function and $R_{0}(t)=\exp \left(-H_{0}(t)\right)$ is the baseline reliability function.

The baseline hazard function of a Weibull distribution is given by $h_{0}(t)=\lambda_{0} \alpha t^{\alpha-1}$, where $\lambda_{0}$ is called the intrinsic failure rate and $\alpha$ is the shape parameter of Weibull distribution. By Eq. (1), the hazard function of Weibull distribution can be expressed as $h(t, \mathbf{x} ; \boldsymbol{\beta})=\lambda_{0} \alpha t^{\alpha-1} e^{\mathbf{x}^{\prime} \boldsymbol{\beta}}$ and, by Eq. (2), its cumulative hazard function is as $H(t, \mathbf{x})=\lambda_{0} t^{\alpha} e^{\mathbf{x}^{\prime} \beta}$.

\section{GLM for right-censored failure time data}

With the proportional hazard assumption, the failure density function is given by

$$
f(t)=h(t) R(t)=h_{0}(t) e^{\mathbf{x}^{\prime} \boldsymbol{\beta}}\left(R_{0}(t)\right)^{e^{\mathbf{x}^{\prime} \beta}} .
$$

For a failure time data set that includes right-censored survival times, each observation can be expressed as a pair $\left(t_{i}, c_{i}\right), i=1,2, \ldots, n$, where $t_{i}$ is either a failure time or censoring time and $c_{i}$ is an indicator variable, which is 1 if the $i^{\text {th }}$ unit failed and 0 if it has not failed. Thus, the likelihood function is given by

$$
L=\prod_{i=1}^{n}\left(f\left(t_{i}\right)\right)^{c_{i}}\left(R\left(t_{i}\right)\right)^{1-c_{i}}=\prod_{i=1}^{n}\left(h\left(t_{i}\right)\right)^{c_{i}} R\left(t_{i}\right) .
$$


From Eqs. (1) and (3) the log-likelihood function can be written as

$$
\ln L=\sum_{i=1}^{n}\left[c_{i} \ln h\left(t_{i}\right)+\ln R\left(t_{i}\right)\right]=\sum_{i=1}^{n}\left[c_{i}\left(\ln h_{0}\left(t_{i}\right)+\mathbf{x}_{i}^{\prime} \boldsymbol{\beta}\right)+e^{\mathbf{x}_{i}^{\prime} \boldsymbol{\beta}} \ln R_{0}\left(t_{i}\right)\right] .
$$

Let $\mu_{i}=\exp \left(\mathbf{x}_{i}{ }^{\prime} \boldsymbol{\beta}\right)\left(-\ln R_{0}\left(t_{i}\right)\right)$, we have

$$
\ln L=\sum_{i=1}^{n}\left[c_{i} \ln h_{0}\left(t_{i}\right)-c_{i} \ln \left(-\ln R_{0}\left(t_{i}\right)\right)+c_{i} \ln \mu_{i}-\mu_{i}\right] .
$$

Note that the last two terms of sum on the right-hand side of Eq. (4) are the same as the kernel of the log-likelihood function of $n$ independent Poisson distributed random variables with mean $\mu_{i}$. The first two terms do not depend on the parameter $\beta$. Therefore, the maximum likelihood estimator $\hat{\boldsymbol{\beta}}$ of (4) is similar to the estimator that maximizes the log-likelihood function of Poisson distributions. If the indicator variable $c_{i}$ is treated as from a Poisson distribution with mean $\mu_{i}$, then the GLM formulation becomes

- The response variables, $c_{i}$ 's, are independently sampled from $\operatorname{Poisson}\left(\mu_{i}\right)$;

- The linear predictor is $\eta_{i}=\mathbf{x}_{i}{ }^{\prime} \boldsymbol{\beta}$;

- The link function is given by $\ln \mu_{i}=\eta_{i}+$ an offset term.

This offset term in the link function is $\ln H_{0}\left(t_{i}\right)$, the log transformation of the baseline cumulative hazard function. This GLM formulation is applicable for any failure time distribution with rightcensored data, as long as the PH assumption holds.

Since the log link function is the canonical link function for the Poisson distribution, the asymptotic variance-covariance matrix of $\hat{\boldsymbol{\beta}}$ is given by

$$
\operatorname{Var}(\hat{\boldsymbol{\beta}})=\left(\mathbf{X}(\xi)^{\prime} \mathbf{W} \mathbf{X}(\xi)\right)^{-1}
$$

where $\mathbf{W}=\operatorname{diag}\left\{\sigma_{i}^{2}\right\}$ and $\sigma_{i}^{2}$ is the variance of Poisson distribution; i.e., $\sigma_{i}^{2}=\mu_{i}=e^{\mathbf{x}_{i}^{\prime} \beta} H_{0}\left(t_{i}\right)$. We replace $t_{i}$ with its expectation, which is given by

$$
E\left[t_{i}\right]=P\left(t<t_{c}\right) \cdot E\left[t_{i} \mid t<t_{c}\right]+P\left(t \geq t_{c}\right) \cdot E\left[t_{i} \mid t \geq t_{c}\right] .
$$

Monroe et al. (2011) has shown that

$$
\mu_{i}=\left[1-e^{-H\left(t_{c}, \mathbf{x}_{i}\right)}\right]=\Phi\left(t_{c}, \mathbf{x}_{i}\right),
$$

where $\Phi$ is the failure time distribution and $t_{c}$ is the censoring time.

For a Weibull distribution with the known shape parameter $\alpha$, it follows that

$$
\mu_{i}=\left[1-\exp \left(-\lambda_{0} t_{c}^{\alpha} e^{\mathbf{x}_{i}{ }^{\prime} \boldsymbol{\beta}}\right)\right]=\left[1-\exp \left(-e^{\beta_{0}+\mathbf{x}_{i}^{\prime} \boldsymbol{\beta}} t_{c}^{\alpha}\right)\right],
$$

where $\beta_{0}=\ln \lambda_{0}$ which plays a role of the intercept term in the linear predictor.

\section{GLM for interval-censored failure time data}

For interval-censored data, the whole testing period is divided into multiple time intervals such as $\left[0, t_{1}\right),\left[t_{1}, t_{2}\right), \cdots,\left[t_{k-1}, t_{k}\right),\left[t_{k}, \infty\right)$, and failures are expected to occur within one of these intervals. Define the failure probability of the $i^{\text {th }}$ test unit within the $j^{\text {th }}$ interval to be

$$
p_{i j}=P\left(t_{j-1} \leq T_{i}<t_{j}\right),
$$

and the conditional probability of surviving at the beginning of the $j^{\text {th }}$ interval but failing within the $j^{\text {th }}$ interval as

$$
\pi_{i j}=P\left(t_{j-1} \leq T_{i}<t_{j} \mid T_{i} \geq t_{j-1}\right), \quad j=1,2, \ldots, k+1 .
$$

It can be shown that $p_{i 1}=\pi_{i 1}$ and $p_{i j}=\left(1-\pi_{i 1}\right)\left(1-\pi_{i 2}\right) \cdots\left(1-\pi_{i, j-1}\right) \pi_{i j}$ for $j=2,3, \ldots, k+1$.

Define an indicator variable, $r$, such that $r_{i j}=0$ if the $i^{\text {th }}$ test unit does not fail within the $j^{\text {th }}$ interval and $r_{i j}=1$ if the $i^{\text {th }}$ test unit does fail. Suppose that there are $n$ items, then the number of observations is $n \times(k+1)$. For example,

$$
\left(0, t_{1}, r_{i 1}\right),\left(t_{1}, t_{2}, r_{i 2}\right), \cdots,\left(t_{k}, \infty, r_{i, k+1}\right), i=1,2, \ldots, n
$$


The likelihood function can be expressed as

$$
L=\prod_{i=1}^{n} \prod_{j=1}^{k+1} p_{i j}^{r_{i j}}
$$

which is equivalent to

$$
L=\prod_{i=1}^{n} \prod_{j=1}^{k+1} \pi_{i j}^{r_{i j}}\left(1-\pi_{i j}\right)^{s_{i j}},
$$

where $s_{i j}=r_{i, j+1}+r_{i, j+2}+\cdots+r_{i, k+1}$. Therefore, $s_{i j}=1$ if the failure of the $i^{\text {th }}$ test unit occurs at a time after the $j^{\text {th }}$ interval and $s_{i j}=0$ if the failure of the $i^{\text {th }}$ test unit occurs within or before the $j^{\text {th }}$ interval.

The likelihood function of Eq. (5) has the same form as the likelihood function of independent binomial random variables. We treat $r_{i j}$ as a binomial random variable with probability $\pi_{i j}$ and sample size $m_{i j}=r_{i j}+s_{i j}$. The data set can be presented as a series of quadruplets:

$$
\left(0, t_{1}, r_{i 1}, m_{i 1}\right),\left(t_{1}, t_{2}, r_{i 2}, m_{i 1}\right), \ldots,\left(t_{k}, \infty, r_{i, k+1}, m_{i, k+1}\right), i=1,2, \ldots, n .
$$

Now, we examine the probability $\pi_{i j}$. Notice that

$$
1-\pi_{i j}=P\left(T_{i} \geq t_{j} \mid T_{i} \geq t_{j-1}\right)=\frac{R\left(t_{j}\right)}{R\left(t_{j-1}\right)} .
$$

By Eq. (3) it becomes

$$
1-\pi_{i j}=\left[\frac{R_{0}\left(t_{j}\right)}{R_{0}\left(t_{j-1}\right)}\right]^{e^{\mathbf{x}_{i}{ }^{\prime} \beta} .}
$$

Applying the natural logarithm function twice yields

$$
\ln \left[-\ln \left(1-\pi_{i j}\right)\right]=\mathbf{x}_{i}^{\prime} \boldsymbol{\beta}+\ln \left[\ln R_{0}\left(t_{j-1}\right) / R_{0}\left(t_{j}\right)\right] .
$$

The second term of the right hand side of Eq. (7) does not depend on the regression coefficient $\beta$, thus Eq. (7) is a complementary log-log link function with an offset term. We can treat $r_{i j}$ 's as independent random variables that follow a binomial distribution with the probability parameter $\pi_{i j}$ and sample size $m_{i j}$, and the GLM formulation is written as

- The response variables, $r_{i j}$ 's, are distributed as independent $\operatorname{Binomial}\left(m_{i j}, \pi_{i j}\right)$;

- The linear predictor is $\eta_{i}=\mathbf{x}_{i}^{\prime} \boldsymbol{\beta}$;

- The link function is given by $\ln \left[-\ln \left(1-\pi_{i j}\right)\right]=\eta_{i}+$ an offset term.

Since the log-log link is not a canonical link for the binomial distribution, we need to introduce $\Delta=\operatorname{diag}\left\{d \theta_{i} / d \eta_{i}\right\}$ in the weight matrix where $\theta_{i}$ is the natural location parameter of the binomial distribution; i.e.,

$$
\begin{aligned}
\Delta & =\operatorname{diag}\left\{\frac{d \theta_{i}}{d \eta_{i}}\right\} \\
& =\operatorname{diag}\left\{\frac{d\left(\ln \frac{\pi_{i j}}{1-\pi_{i j}}\right)}{d\left(\ln \left(-\ln \left(1-\pi_{i j}\right)\right)\right.}\right\} \\
& =\operatorname{diag}\left\{-\frac{\ln \left(1-\pi_{i j}\right)}{\pi_{i j}}\right\} .
\end{aligned}
$$

Then, the asymptotic variance-covariance matrix of $\hat{\beta}$ is given by

$$
\begin{aligned}
\operatorname{Var}(\hat{\boldsymbol{\beta}}) & =\left(\mathbf{X}^{*}(\xi)^{\prime} \mathbf{W} \mathbf{X}^{*}(\xi)\right)^{-1} \\
& =\left(\mathbf{X}^{*}(\xi)^{\prime} \Delta \mathbf{V} \Delta \mathbf{X}^{*}(\xi)\right)^{-1},
\end{aligned}
$$

where $\mathbf{X}^{*}(\xi)=\mathbf{X}(\xi) \otimes \mathbf{1}_{k+1}$ and $\mathbf{V}=\operatorname{diag}\left\{\sigma_{i j}^{2}\right\}$. Note that, instead of using $\mathbf{X}(\xi)$, the original model matrix, $\mathbf{X}^{*}(\xi)$, which is a matrix of size $n(k+1) \times p$, is used. Each row of $\mathbf{X}(\xi)$ is repeated $(k+1)$ times in $\mathbf{X}^{*}(\xi)$ because each test unit has $(k+1)$ intervals. 
In a binomial distribution, $\sigma_{i j}^{2}=m_{i j} \pi_{i j}\left(1-\pi_{i j}\right)$, which includes the random variable $m_{i j}$. Replacing $m_{i j}$ with its expectation, the weight matrix becomes

$$
\begin{aligned}
\mathbf{W} & =\Delta \mathbf{V} \Delta \\
& =\operatorname{diag}\left\{\left(-\frac{\ln \left(1-\pi_{i j}\right)}{\pi_{i j}}\right) E\left(m_{i j}\right) \pi_{i j}\left(1-\pi_{i j}\right)\left(-\frac{\ln \left(1-\pi_{i j}\right)}{\pi_{i j}}\right)\right\} \\
& =\operatorname{diag}\left\{\frac{\left\{\ln \left(1-\pi_{i j}\right)\right\}^{2}\left(1-\pi_{i j}\right)}{\pi_{i j}} E\left(m_{i j}\right)\right\} .
\end{aligned}
$$

Assuming a Weibull distribution for a product's lifetime, we have $R(t, \mathbf{x})=\exp (-H(t, \mathbf{x}))=$ $\exp \left(-\lambda_{0} t^{\alpha} e^{\mathbf{x}^{\prime} \boldsymbol{\beta}}\right)=e^{-t^{\alpha} e^{\beta_{0}+\mathbf{x}^{\prime} \boldsymbol{\beta}}}$. Substituting it into (6) yields

$$
1-\pi_{i j}=\frac{e^{-t_{j}^{\alpha} e^{\beta_{0}+x^{\prime} \beta}}}{e^{-t_{j-1}^{\alpha} e^{\beta_{0}+x^{\prime} \beta}}} .
$$

Assume all time intervals have the same length, $\Delta t$. Then,

$$
1-\pi_{i j}=\frac{e^{-(j \Delta t)^{\alpha} e^{\beta_{0}+x^{\prime} \beta}}}{e^{-((j-1) \Delta t)^{\alpha} e^{\beta_{0}+x^{\prime} \beta}}}=e^{\left((j-1)^{\alpha}-j^{\alpha}\right) \Delta t^{\alpha} e^{\beta_{0}+x^{\prime} \beta} .}
$$

We also have

$$
\begin{aligned}
E\left(m_{i j}\right) & =0 \times P\left(T_{i}<t_{j-1}\right)+1 \times P\left(T_{i} \geq t_{j-1}\right) \\
& =P\left(T_{i} \geq t_{j-1}\right) \\
& =R\left(t_{j-1}, \mathbf{x}_{i}\right) \\
& =e^{-((j-1) \Delta t)^{\alpha} e^{\beta_{0}+\mathbf{x}_{i}{ }^{\prime} \beta}} .
\end{aligned}
$$

Substituting Eq. (9) and Eq. (10) into Eq. (8) yields the weight matrix for the interval-censored Weibull failure time data:

$$
\begin{aligned}
& \mathbf{W}=\operatorname{diag}\left\{\frac{\left\{\left((j-1)^{\alpha}-j^{\alpha}\right) \Delta t^{\alpha} e^{\beta_{0}+\mathbf{x}_{i}^{\prime} \boldsymbol{\beta}}\right\}^{2} e^{\left((j-1)^{\alpha}-j^{\alpha}\right) \Delta t^{\alpha} e^{\beta_{0}+\mathbf{x}^{\prime} \beta}}}{1-e^{\left((j-1)^{\alpha}-j^{\alpha}\right) \Delta t^{\alpha} e^{\beta_{0}+x^{\prime} \beta}}} e^{-((j-1) \Delta t)^{\alpha} e^{\beta_{0}+\mathbf{x}_{i}{ }^{\prime} \beta}}\right\} \\
& =\operatorname{diag}\left\{\frac{\left((j-1)^{\alpha}-j^{\alpha}\right)^{2} \Delta t^{2 \alpha} e^{2\left(\beta_{0}+\mathbf{x}_{i}^{\prime} \beta\right)-j^{\alpha} \Delta t^{\alpha} e^{\beta_{0}+\mathbf{x}_{i}{ }^{\prime} \beta}}}{1-e^{\left((j-1)^{\alpha}-j^{\alpha}\right) \Delta t^{\alpha} e^{\beta_{0}+\mathbf{x}_{i}{ }^{\prime} \beta}}}\right\} \text {. }
\end{aligned}
$$

\section{Introduction to the package ALTopt}

The main purpose of the ALTopt package is to construct $D-, U-$, and $I$-optimal ALT test plans. Two main functions, al topt.rc and altopt.ic, are developed respectively for the right-censoring and interval-censoring cases. The following assumptions are required for using this package:

- Failure time data follows the Weibull distribution where the shape parameter is specified by the user.

- Log-linear functions are used to model the relationship between a failure time distribution parameter and the stress factors.

- For interval-censored data, all the intervals have the same length.

Lastly, the package can accommodate many stress factors, but 5 or fewer is recommended for computational efficiency.

This package also provides two functions for evaluating existing test plans - alteval.rc and alteval.ic. These functions can be used for comparing test plans generated by this ALTopt package or any other methods. Graphical displays of prediction variance are made available. These plotting features enhance the usefulness of this package for comparing and selecting test plans.

\section{Creating optimal ALT test plans}

The syntax of altopt.rc and altopt.ic functions are as follows: 
altopt.rc(optType, N, tc, nf, alpha, formula, coef,

useCond, useLower, useUpper, nOpt $=1, \mathrm{nKM}=30, \mathrm{nCls}=\mathrm{NULL}$ )

altopt.ic(optType, N, t, k, nf, alpha, formula, coef,

useCond, useLower, useUpper, nOpt $=1, \mathrm{nKM}=30, \mathrm{nCls}=\mathrm{NULL}$ )

The arguments within these functions are:

- optType - Choice of "D", "U", and "I" optimality.

- $\mathrm{N}$ - The number of test units.

- tc (altopt.rc only) - The planned right censoring time.

- $t$ (al topt .ic only) - The planned total testing time (i.e., the end point of the last interval).

- $k$ (altopt. ic only) - The number of time intervals.

- $\mathrm{nf}-$ The number of stress factors.

- alpha - The value of the shape parameter of the Weibull distribution.

- formula - The object of class "formula" expressing the linear predictor model.

- coef - The numeric vector containing the coefficients of each term in formula.

- useCond - The numeric vector of use condition. It should be provided when optType is "U". The length of the vector should be same as the number of stress factors.

- useLower - The numeric vector of lower bound of use region in coded units. It should be provided when optType is "I". The length of the vector should be the same as the number of stress factors.

- useUpper - The numeric vector of upper bound of use region in coded units. It should be provided when optType is "I". The length of the vector should be the same as the number of stress factors.

- nopt - The number of repetitions of optimization processes. The default value is 1 . If nopt is larger than 1, each optimization process starts from randomly chosen design points in the design region and each solution may slightly differ. The output shows the best solution overall.

- nKM - The number of repetitions of k-means clustering, which is used to generate the optimal design clustered by kmeans. The default value is 30 .

- $\mathrm{nCls}$ - The number of clusters used for k-means clustering. If not specified, it is set as the number of parameters in the linear predictor model.

We use the function stats: : optim with the "L-BFGS-B" method to perform optimization. This function allows box constraints on design variables. In our case, we have a cuboidal design region where the levels of each stress factor are coded to be between 0 and 1 . More details about the "L-BFGS-B" method are available in Byrd et al. (1995).

The output of these functions are given as a list with the following components:

- call - The matched call.

- opt. design. rounded - The optimal design clustered by rounding.

- opt.value. rounded - The objective function value of opt. design. rounded.

- opt. design. kmeans - The optimal design clustered by k-means algorithm.

- opt.value.kmeans - the objective function value of opt. design. kmeans.

The procedure begins by generating an initial test plan with $N$ design points, which are randomly selected from possible points in the design region. For example, if we have 100 test units and 2 stress factors the optimization process begins from 100 randomly chosen initial points, which spread out over the design region. Throughout the optimization procedure, each of these 100 points converges to its own optimal location. to create a practical test plan, it is sometimes necessary to enforce some clustering procedure to reduce the number of distinct design points. Two clustering methods are implemented in the package. When the design points are very close, the simple method of rounding (to the $3^{\text {rd }}$ decimal place) the stress values is effective and straightforward. When there are still too many design points, the alternative is to use k-means clustering, which requires the specification of the number of clusters, $\mathrm{nCls}$. By carefully selecting the number of clusters, it is possible to reduce the number of distinct design points without significantly affecting the value of the objective function. The final recommended test plans are provided by the elements, opt.design. rounded, or opt.design.kmeans, presented by a table containing each design point location and the number of test units allocated at each design point. The corresponding values of the objective function of these plans are also stored in opt.value. rounded and opt.value. kmeans. 
The design. plot function displays the recommended test plan as a bubble plot on a two-dimensional design region of user-specified stress factors. The size of each bubble represents the relative size of the test unit allocations. The arguments of design. plot are as follows:

design.plot(design, xAxis, yAxis)

- design - A data frame containing the coordinates and the test unit allocation at each design point. The components, opt. design. rounded or opt.design. kmeans, of an output created by altopt.rc or altopt.ic can be given for this argument directly and any other design with the same form of those can also be given.

- xAxis - The name of the factor to be displayed on the $x$ axis.

- yAxis - The name of the factor to be displayed on the $y$ axis.

\section{Evaluating ALT test plans}

This package provides several methods to evaluate an ALT test plan. The first method is the numerical evaluation of a given test plan using al teval. rc or alteval.ic. These functions return the value of the objective function of the test plan. The arguments are as follows:

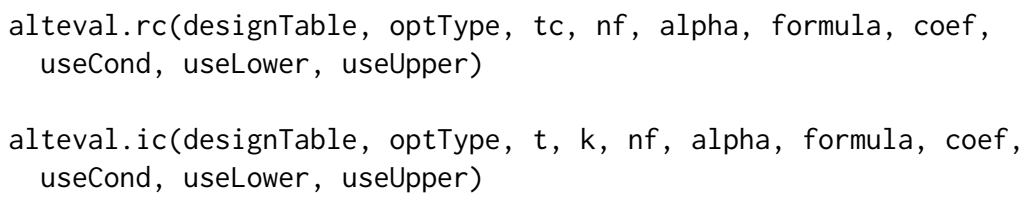

The existing test plan is specified in the argument designTable. The other arguments of al teval.rc and alteval.ic are similar to the arguments in al topt.rc and al topt.ic.

ALTopt also provides three different graphs for evaluating a test plan - the prediction variance (PV) contour plot, the fraction of use space (FUS) plot, and the variance dispersion of use space (VDUS) plot. These graphical tools are useful when visualizing the prediction variance throughout the entire use-space region (Myers et al., 2009, chap. 8). The PV contour plot displays the contours of the estimated prediction variance from the design region to the use region of a two-dimensional userspecified stress factor space. Functions pv. contour. rc and pv. contour. ic generate the PV contour plot of an ALT test plan with right and interval censoring, respectively. The FUS plot is an extension of the fraction of design space (FDS) proposed by Zahran et al. (2003). The vertical axis of a FUS plot is the fraction of the use space region that has prediction variance less than or equal to the given values in the horizontal axis. Functions pv. fus.rc and pv. fus.ic create the FUS plot of right and interval censoring ALT plans, respectively. In addition, the FUS curves of multiple designs can be overlaid on one graph by using compare. fus, so these designs can be compared graphically. The VDUS plot is an extension of the variance dispersion graphs (VDGs) of Giovannitti-Jensen and Myers (1989) to the cuboidal use space region. It shows plots of minimum, average and maximum prediction variance from the center to the boundary of the use region. The comparison of multiple VDUS is also available through compare.vdus. The arguments of these functions are omitted here, because they are similar to previously described functions.

\section{An example with two stress factors and right censoring}

In this section, we demonstrate the use of ALTopt using the right-censored ALT data set from Yang and Pan (2013). In this experiment, an ALT of 100 test units is conducted with two stress factors temperature and humidity. The lowest and highest stress levels in the test region are $\left(60{ }^{\circ} \mathrm{C}, 60 \%\right)$ and $\left(110{ }^{\circ} \mathrm{C}, 90 \%\right)$, respectively. The normal use condition is $\left(30^{\circ} \mathrm{C}, 25 \%\right)$, while the typical use region has the range from $\left(20^{\circ} \mathrm{C}, 20 \%\right)$ to $\left(40^{\circ} \mathrm{C}, 30 \%\right)$. The natural stress variables of these two factors are defined by $S_{1}=11605 / T$, where $T$ is the temperature in degrees Kelvin (i.e., temp ${ }^{\circ} \mathrm{C}+273.15$ ), and $S_{2}=\ln (h)$, where $h$ is the relative humidity. These values are assigned to the following variables:

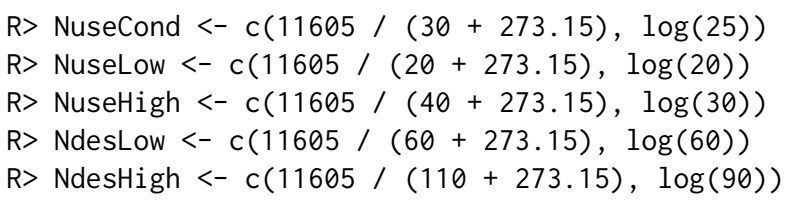


Next, we apply a coding scheme on these natural variables so that the highest stress level becomes $(0$, $0)$ and the lowest stress level becomes $(1,1)$.

$$
x_{1}=\frac{S_{1}-S_{1}^{H}}{S_{1}^{L}-S_{1}^{H}}, \quad x_{2}=\frac{S_{2}-S_{2}^{H}}{S_{2}^{L}-S_{2}^{H}} .
$$

Here, $x_{1}$ and $x_{2}$ are the coded stress variables of $S_{1}$ and $S_{2}$, respectively. The ALTopt package provides a utility function, convert. stress. level, to convert the natural stress level to the coded stress level, and vice versa. The use condition and the use stress region are accordingly coded as follows:

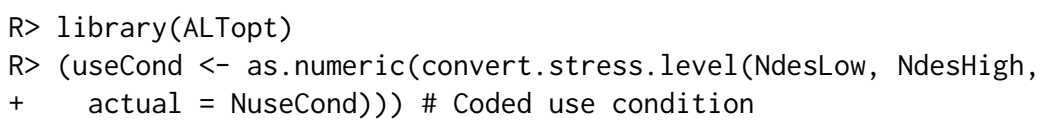

[1] $1.758337 \quad 3.159172$

R> (useLower <- as.numeric(convert.stress.level(NdesLow, NdesHigh,

+ actual $=$ NuseHigh $))$ ) \# Coded use region's lower bound

[1] 1.4894142 .709511

R> (useUpper <- as.numeric(convert.stress.level(NdesLow, NdesHigh,

$+\quad$ actual $=$ NuseLow)) ) \# Coded use region's upper bound

[1] $2.045608 \quad 3.709511$

We assume that the failure times follow an exponential distribution, i.e., alpha $=1$, and the prespecified linear predictor is given by

$$
\eta_{i}=-4.086 x_{1}-1.476 x_{2}+0.01 x_{1} x_{2} .
$$

Suppose the total testing time is 30 time units. The D-optimal test plan is generated by the following lines of code:

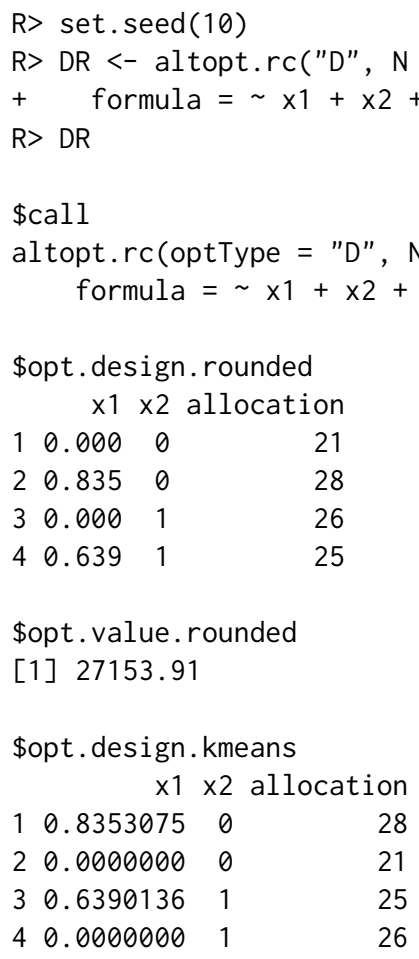

\$opt.value.kmeans

[1] 27153.92

While the formula does not include the intercept term explicitly, the value of the intercept parameter still needs to be specified (in this case, it is 0 ). From the final design output, we noticed that the designs generated by rounding and clustering are almost the same.

We can also generate the $U$-optimal and $I$-optimal designs using the following lines of code: 


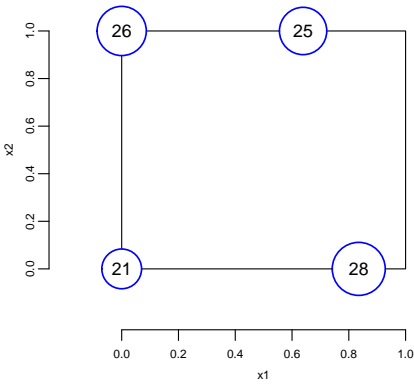

(a)

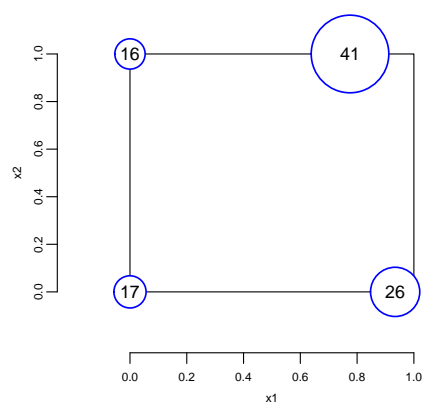

(b)

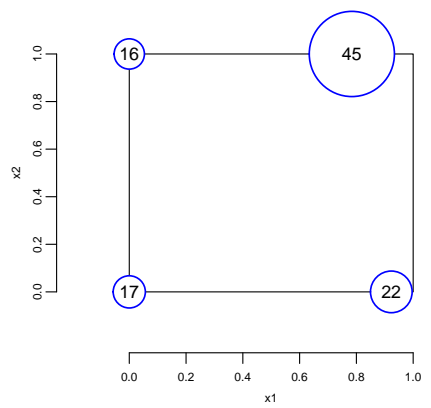

(c)

Figure 1: Design plots of (a) D-optimal, (b) U-optimal and (c) I-optimal designs with right censoring drawn by design. plot function.

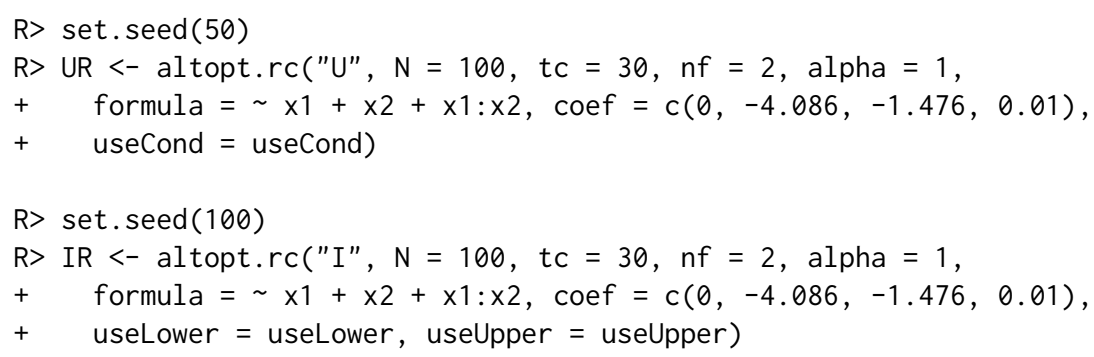

Using design. plot function, we can draw the bubble plots of these test plans.

$R>$ design.plot(DR\$opt.design.rounded, $x A x i s=x 1, y A x i s=x 2)$

$R>$ design.plot(UR\$opt.design.rounded, $x A x i s=x 1, y A x i s=x 2$ )

$\mathrm{R}>$ design.plot(IR\$opt.design.rounded, $x$ Axis $=x 1$, yAxis $=x 2$ )

From Figure 1, one can see that the $U$ - and $I$-optimal test plans resemble each other, while the $D$ optimal test plan is very different from the other two. This is expected because the objective functions of $U$ - and I-optimal designs involve the variance of reliability prediction, while the $D$-optimal design involves the variance of parameter estimation. From the $U$ - and $I$-optimal test plans, it is noticeable that a large number of test units is allocated at the lowest stress level. This type of test unit allocation scheme is common in ALTs (e.g., Meeker and Nelson, 1975).

To compare the $D$-optimal test plan and the $U$-optimal test plan, the pv.contour.rc function generates the contour plot of prediction variance using the following lines of code:

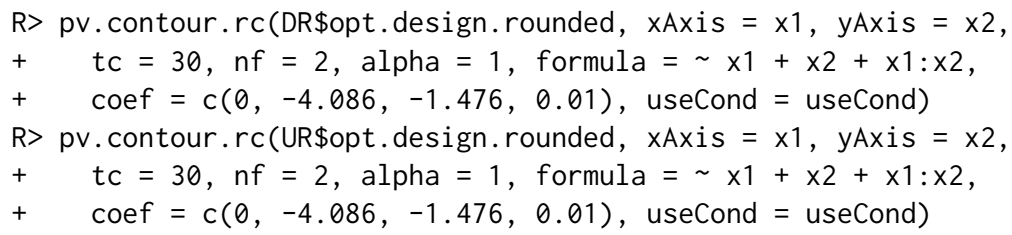

Figure 2 shows that the $U$-optimal test plan has lower prediction variance than the $D$-optimal test plan at the normal use condition. The FUS and VDUS plots can also be used for further comparison of these test plans. These plots are shown in Figure 3.

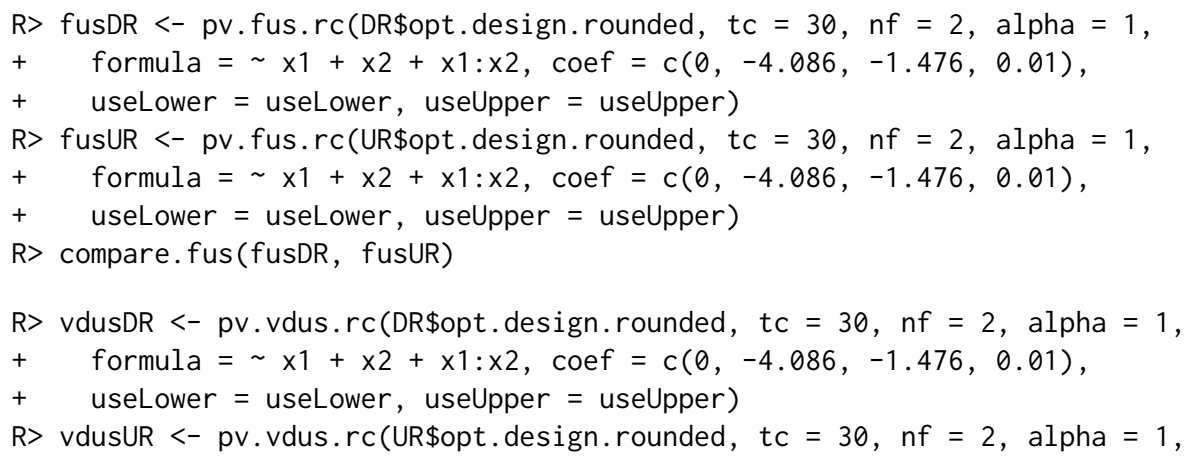




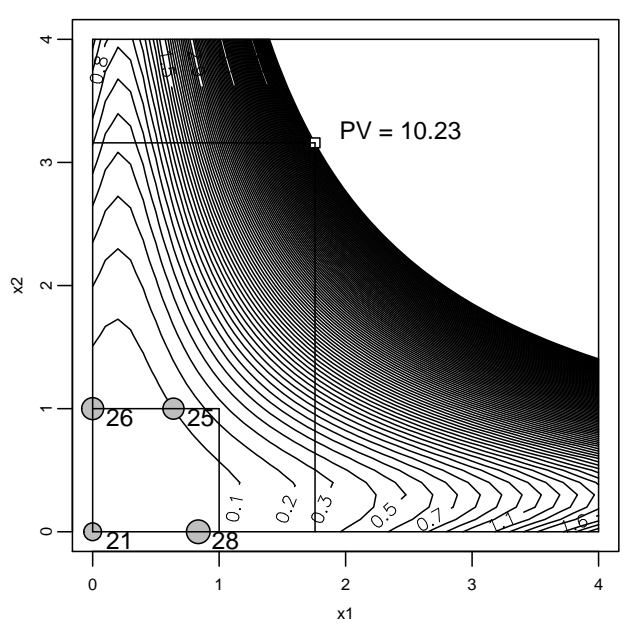

(a)

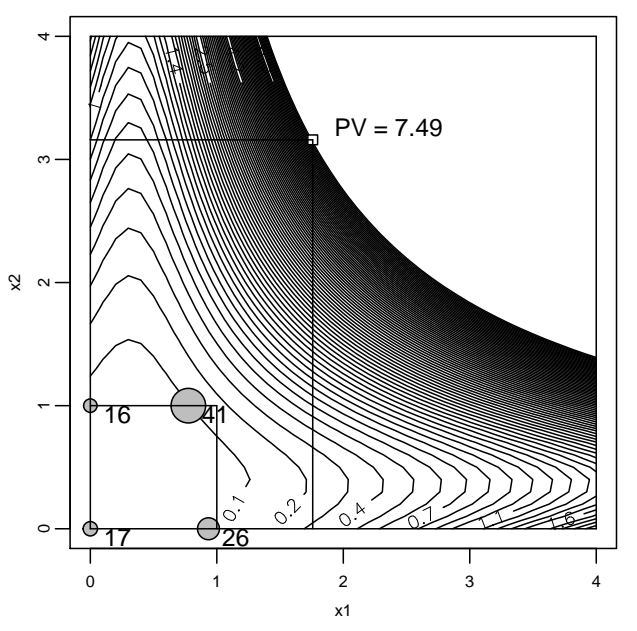

(b)

Figure 2: Prediction variance contour plots of (a) D-optimal and (b) U-optimal designs with right censoring drawn by pv. contour. rc function.

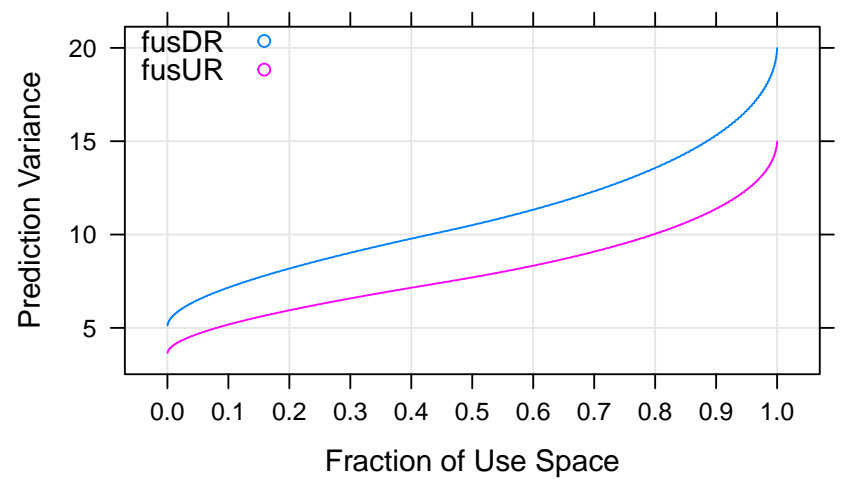

(a)

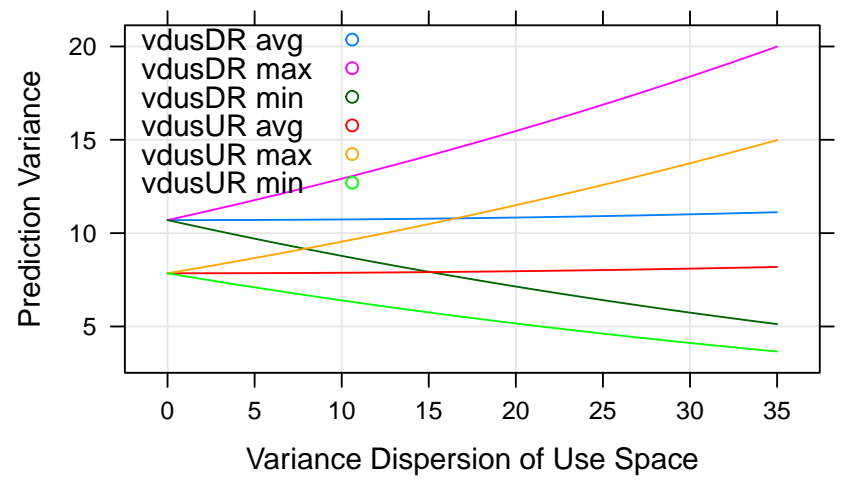

(b)

Figure 3: Comparison of D-optimal and U-optimal designs with right censoring using (a) FUS plot and (b) VDUS plot. 
+ formula $=\sim x 1+x 2+x 1: x 2$, coef $=c(0,-4.086,-1.476,0.01)$,

+ useLower $=$ useLower, useUpper $=$ useUpper $)$

$\mathrm{R}>$ compare.vdus (vdusDR, vdusUR)

Figure 3 shows that the $U$-optimal test plan performs better in majority of the use-space region with respect to the prediction variance.

Finally, the convert. stress. level function is useful for converting the $U$-optimal test plan to the natural stress level conditions.

R> convert.stress.level(NdesLow, NdesHigh, stand = UR\$opt.design. rounded)

$\begin{array}{rrrr} & \times 1 & \times 2 & \text { allocation } \\ 1 & 30.28840 & 4.499810 & 17 \\ 2 & 34.53414 & 4.499810 & 26 \\ 3 & 30.28840 & 4.094345 & 16 \\ 4 & 33.81136 & 4.094345 & 41\end{array}$

\section{Summary}

This paper describes the ALTopt package in R for constructing optimal ALT test plans for right- and interval-censored data. The package accommodates three statistical optimality criteria - $D$-optimal, $U$-optimal and I-optimal. It applies the GLM approach to the modeling of failure/censoring times and the derivation of the asymptotic variance-covariance matrix of regression coefficients. Failure times are assumed to follow a Weibull distribution. To use the package effectively, users are required to specify the linear predictor of the GLM and the shape parameter of the Weibull distribution. An example demonstrated the construction of optimal test plans for an ALT with two stress factors and right-censored data. This package also provides graphical functions for evaluating and comparing various test plans.

\section{Bibliography}

R. H. Byrd, P. Lu, J. Nocedal, and C. Zhu. A limited memory algorithm for bound constrained optimization. SIAM Journal on Scientific Computing, 16(5):1190-1208, 1995. [p182]

A. Giovannitti-Jensen and R. H. Myers. Graphical assessment of the prediction capability of response surface designs. Technometrics, 31(2):159-171, 1989. [p183]

R. T. Johnson and D. C. Montgomery. Choice of second-order response surface designs for logistic and Poisson regression models. International Journal of Experimental Design and Process Optimisation, 1(1): 2-23, 2009. [p178]

W. Q. Meeker and L. A. Escobar. Statistical Methods for Reliability Data, volume 314. John Wiley \& Sons, 1998. [p178]

W. Q. Meeker and W. Nelson. Optimum accelerated life-tests for the Weibull and extreme value distributions. IEEE Transactions on Reliability, 24(5):321-332, 1975. [p185]

E. M. Monroe, R. Pan, C. M. Anderson-Cook, D. C. Montgomery, and C. M. Borror. A generalized linear model approach to designing accelerated life test experiments. Quality and Reliability Engineering International, 27(4):595-607, 2011. [p177, 179]

R. H. Myers, D. C. Montgomery, and C. M. Anderson-Cook. Response Surface Methodology: Process and Product Optimization Using Designed Experiments, volume 705. John Wiley \& Sons, 2009. [p183]

W. B. Nelson. A bibliography of accelerated test plans. IEEE Transactions on Reliability, 54(2):194-197, 2005a. [p177]

W. B. Nelson. A bibliography of accelerated test plans Part II-references. IEEE Transactions on Reliability, 54(3):370-373, 2005b. [p177]

K. Seo and R. Pan. ALTopt: Optimal Experimental Designs for Accelerated Life Testing, 2015. URL https://CRAN.R-project. org/package=ALTopt. R package version 0.1.1. [p177]

T. Yang and R. Pan. A novel approach to optimal accelerated life test planning with interval censoring. IEEE Transactions on Reliability, 62(2):527-536, 2013. [p177, 183] 
A. Zahran, C. M. Anderson-Cook, and R. H. Myers. Fraction of design space to assess prediction capability of response surface designs. Journal of Quality Technology, 35(4):377-386, 2003. [p183]

Kangwon Seo

School of Computing, Informatics, Decision Systems Engineering

Arizona State University

699 S Mill Ave, Tempe, AZ 85281

USA

kseo7@asu.edu

Rong Pan

School of Computing, Informatics, Decision Systems Engineering

Arizona State University

699 S Mill Ave, Tempe, AZ 85281

USA

rong. pan@asu. edu 